tionen. So verwandelt sich Chlorblei darin in braunes Bleisuperoxyd, und Manganchlorür in Superoxyd, während frisches Chlorwasser diese Wirkung nicht auf jene Körper ausibt. Millo n hat bei einer Untersuchung, um sich zu überzeugen, welcher Chlorverbindung jene Eigenschaft zukomme, gefunden, dass diese ausschliesslich die unterchlorige Säure ist. Man kann diese Säure selbst in Chlorlösungen und bei sehr geringen Quantitäten durch eben diese beiden Reagention, und namentich durch Manganchlorür entdecken.

Die Wirkungsweise des Chlors auf Wasser, wobei die unterchlorige Säure entsteht, ist offenbar dieselbe, welche bei anderen wasserstoffhalligen Körpern stalt findet. Das Chlor vertritt den Wasserstoff in seinen Verbindungen. und wenn diese Reactionen, wie hier, gewisse Grenzen haben, so liegt dieses darin begrundet, dass die unterchlorige Säure wiederum durch die gleichzeitig aufretende Salzsaure zersetzt wird. Wie Versuche Millo n's gezeigt haben, können unterchlorige Säure und Salzsaure nur dann neben einander existiren, wenn sie in sehr vielem Wasser gelöst sind.

Das einfache Verhältniss, das zwischen Salzsäure und unterchloriger Säure existirt, muss man nach Millon bei der Bestimmung der Zusammensetzung des Chlorschwefels in Anschlag bringen. In diesen Verbindungen muss die Zusammensetzung des Schwefelwasserstoffs zu Grunde gelegt werden; das Chlor vertritt darin dann den Wasserstoff. Demnach ist gewiss anzunehmen, dass der Chlorschwefel mil dem grössten Chlorgehalte aus gleichen Aequivalenten Chlor und Schwefel bestehen muss. Dadurch erscheint es auch natürlich, dass die Bemühungen der Chemiker, einen höher gechlorien Chlorschwefel darzustellen, fruchtlos geblieben sind. Umgekehrt gelang es hingegen, mehr Schwefel mit 1 Aeq. Chlor zu verbinden. Nach dieser Beobachtung wïrden die Chlorschwefel den Polysulphüren des Wasserstoffs correspondiren und sich an die Oxyde in folgender symmetrischer Ordnung anreihen: HO, HS, HS ${ }^{n}$, ClO, CIS, ClS . (Compt.rend. - Pharm. Centrbl. 1819. No. 13.)

$B$.

\title{
Neue Verbindung von Borsäure mit Natron.
}

Beim Vermischen einer Boraxlösung mit einer Salmiaklösung entwickelt sich Ammoniakgas. Diese Thatsache veranlasste B olle y zu nachstehendem Versuche:

Eine Lösung von 1 Aeq. Salmiak und 2 Aeq. Borax 
in Wasser wurde so lange gekocht, als sich noch Ammoniakdämpfe entwickelten. Dies geschah gegen Ende der Operation in sehr geringem Maasse und unterblieb, als die Lösung sehr concentrirt geworden war, ganz, während die wieder verdünnte Lösung aufs Neue Ammoniak entwickelte. Aus der zuletzt filtrirten und abgedampften Lösung krystallisirte, wenn die Temperatur nur irgend hoch war, zuerst Borax, worauf die Lösung syrupartig wurde und keine Krystalle mehr absetzle. Bei einem sehr allmäligen Abdampfen der Lösung dagegen setzlen sich Krystallkrusten ab, die in ihrem äusseren und chemischen Verhalten ubereinstimmten. Sie waren in $\ddot{j}$ bis 6 Theilen Wasser von gewöhnlicher Temperatur auflijslich, und die Auflösung unterschied sich dadurch wesentlich von einer Boraxlösung, dass sie Curcumapapier nich t bräunte und auch $\mathrm{k}$ alt mit verdünnten Säuren vermischt, Borsäure fallen liess. Einer Analyse unterworfen, ergaben clie Krystalle die Zusammensetzung eines Salzes mit der Formel: $\mathrm{NaO}, 4 \mathrm{BO}^{3}+10 \mathrm{HO}$ und waren also das schon lange in der Reihe der drei bekannten boraxsauren Natronsalze, nämlich des $\mathrm{Na}, \mathrm{BO}^{3}$, des $\mathrm{NaO}, 2 \mathrm{BO}^{3}$ und des $\mathrm{NaO}, 6 \mathrm{BO}^{3}$ varmisste vierfach boraxsaure Natronsalz. Offenbar wird bai der Darstellung des vierfach boraxsauren Natrons auf die angegebene Weise von dem Chlor des Salmiaks dem Borax ein Theil seines Natrons entzogen, eine entsprechende Menge Chlornatrium gebildet, Ammoniak dadurch frei gemacht und aus dem Rest des Natrons ein Natronsalz mit grösserem Borsäuregehalt gebildet.

Das Verhalten des Salmiaks zum Borax giebt dem Verfasser Veranlassung, weiter cine der Paye n'sche Hypothese über die Bildung von Borsäure aus Schwefelhor entgegenstehende Erklärungsweise des Vorkommens der im Toscanischen und auf der Insel Volcano in unmittelbarer vulkanischer Nachbarschaft und in Begleitung heisser Wasserdämpfe aus der Erde emporsteigenden Borsäure zu geben. Nachdem er nämlich gefunden, dass sich der Salmiak ähnlich, wie zum Borax, auch zum Borazit und Datolit, zur borsauren Bitter- und Kalkerde verhält. bemerkt er, dass es ausser diesen Mineralien und dem Tinkal noch viele borsäurehaltige, $z$. B. Turmalin, Axinit u. a. m. gebe, und dass es in vulkanischen Gegenden, wo das Vorkommen des natürlichen Salmiaks elwas ganz Gewöhnliches sei, nur des gleichzeitigen Vorkommens eines solchen Minerals bedürfe. um die Bildung der Borsäure zu bedingen. Der Umsland, dass bei uiberschüssigem Salmiak der Borax vollständig in Kochsalz und Borsäure zerlegt 
wird, lässt allerdings erwarten, dass es mit anderen Borsäureverbindungen sich ehen so verhalte, und das von $\mathrm{Pa}$ ye $\mathrm{n}$ selbst wahrgenommene Vorkommen von Ammoniak in den von ihm aufgefangenen borsaurehaltigen Dämpfen am Monte rotondo in Toscana spricht deullich für die Richtigkeit der von B olley gegebenen Erklarungsweise über die Bildung der Borsaure in vulkanischen Gegenden. (Annal. der Chem. u. Pharm. Bd.68. p.122-126) G.

\section{Neue schwefelwasserstoffhaltige Soolquelle.}

Bromeis hat eine Soolquelle, die im Sommer 1847 am westlichen Abhange des Lindener Berges bei llannover erbohrt worden ist, untersucht. Das Wasser derselben hat bei $9^{\circ} \mathrm{C}$. ein spec. Gewicht von 1,0808, besitzt einen starken Geruch nach Schwefelwasserstoff und trübt sich an der Luft. 1000,00 Theile Wasser enthalten:

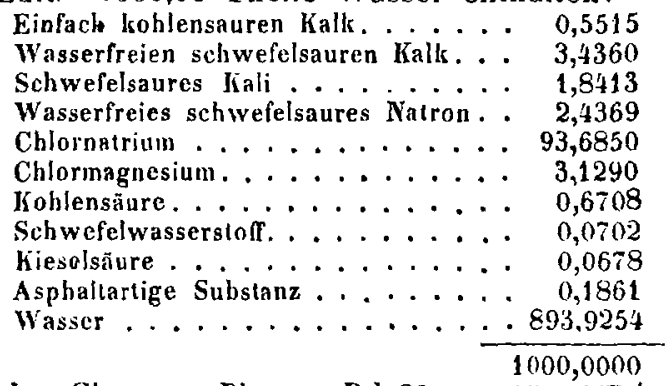

(Annal. der Chem. u. Pharm. Bd.69. p. 115-117.)

G.

Ueber die medicinische Wirkung des Jodkaliums bei Blei- und Quecksilbervergiftungen.

Melsens hat in Gemeinschaft mit Dr. N. Guillot den Vorschlag gemacht, die Metallsubstanzen, welche aus dem Korper fortgeschafft werden sollen, an einen solchen Körper zu binden, der an und für sich schon leicht vom Organismus ausgeschieden wird. Diese Methode der Heilung hat sich bereits bei allen den unlöslichen Verbindungen, welche Quecksilbersalze mit den gewöhnlichen Substanzen im Körper bilden, verwirklicht, sie können durch Jodkalium gelöst und mit demselben schnell aus dem Körper entfernt werden.

Melsens hat nun auch gefunden, dass Jodkalium, in gewisser Weise angewandt, die durch Blei entstandenen 